\title{
La nationalité/citoyenneté italienne : « familiste » mais non «culturelle»?
}

Document Version

Accepted author manuscript

Link to publication record in Manchester Research Explorer

\section{Citation for published version (APA):}

Sredanovic, D. (2018). La nationalité/citoyenneté italienne : « familiste » mais non « culturelle »? Pôle Sud, 47(2), 133-146. https://www.cairn.info/revue-pole-sud-2017-2-page-133.htm

\section{Published in:}

Pôle Sud

\section{Citing this paper}

Please note that where the full-text provided on Manchester Research Explorer is the Author Accepted Manuscript or Proof version this may differ from the final Published version. If citing, it is advised that you check and use the publisher's definitive version.

\section{General rights}

Copyright and moral rights for the publications made accessible in the Research Explorer are retained by the authors and/or other copyright owners and it is a condition of accessing publications that users recognise and abide by the legal requirements associated with these rights.

\section{Takedown policy}

If you believe that this document breaches copyright please refer to the University of Manchester's Takedown Procedures [http://man.ac.uk/04Y6Bo] or contact uml.scholarlycommunications@manchester.ac.uk providing relevant details, so we can investigate your claim.

\section{OPEN ACCESS}




\title{
LA NATIONALITÉ/CITOYENNETÉ ITALIENNE: «FAMILISTE» MAIS NON «CULTURELLE»?
}

\author{
Djordje Sredanovic \\ Université Libre de Bruxelles - GERME/MAM \\ Djordje.Sredanovic@ulb.ac.be
}

Version de l'auteur - dernière version publié en Pôle Sud, 47 : 133-146 -

https://www.cairn.info/revue-pole-sud-2017-2-page-133.htm

Résumé : Ce texte propose l'analyse de la loi sur la nationalité italienne, de son développement et des propositions de réforme de cette loi. L'analyse est complétée par une recherche qualitative sur les conceptions de la nationalité de migrants et ouvriers locaux. Bien que la loi italienne sur la nationalité soit souvent considérée comme "ethnique», l'analyse montre que, si la loi est restrictive envers les immigrés et favorable aux émigrés, les normes explicitement fondées sur l'appartenance ethnique ou la compétence culturelle sont très limitées. Les propositions de loi ont visé à introduire des tests de compétence culturelle pour les immigrés mais ont plus rarement fait référence à l'ethnicité, et les entretiens qualitatifs montrent une conception de la citoyenneté/nationalité économique plutôt que culturelle parmi la population.

Mots-clés : Nationalité, citoyenneté, Italie, ethnicité, naturalisation

Abstract: This text proposes an analysis of the Italian nationality law, of its development and of its proposed reforms. The analysis is completed with a qualitative research on the conceptions of citizenship and nationality among migrants and local factory workers. The Italian nationality law is often considered an "ethnic" law, but the analysis shows that, while the law is restrictive towards immigrants and favourable to the emigrants, there are very few norms in the law that are explicitly based on ethnic belonging or on cultural competence. Law proposals have tried to introduce cultural competence tests for the immigrants, but have made reference more rarely to ethnicity, and 
the qualitative interviews show an economic conception of citizenship and nationality, rather than a cultural one, among the population.

Keywords: Nationality, citizenship, Italy, ethnicity, naturalization

La législation sur la nationalité/citoyenneté ${ }^{1}$ depuis 25 ans a suscité l'intérêt des chercheurs en sciences sociales. Ce champ de recherche a commencé avec l'analyse de Brubaker (1992) sur l'histoire de la législation sur la nationalité en France et en Allemagne, considérée en tant que miroir des conceptions nationales d'appartenance. Le champ s'est ensuite étendu à l'analyse des déterminants institutionnels et politiques (histoire et typologie des migrations, circulation de politiques) de cette législation (Weil et Hansen, 1999), à l'exploration des idées d'appartenance de la population générale à travers les opinions sur la nationalité (citoyenneté quotidienne - MillerIdriss, 2006 ; voir aussi Duchesne, 1997 ; Venel, 2004 ; Ribert, 2006 pour le concept très proche de « citoyenneté ordinaire »), jusqu'à l'analyse de l'interaction de statut légal et capital dans la pratique effective de la citoyenneté (citoyenneté matérielle - Baglioni, 2015).

Dans ce texte, je propose une analyse de la loi italienne sur la nationalité (Loi 91 du 1992), des changements dont elle a fait l'objet, et des propositions de modification de cette loi. J'ai combiné cette analyse institutionnelle «à la Brubaker» avec des données produites dans le cadre d'une recherche qualitative sur la citoyenneté quotidienne en Italie auprès de migrants et d'ouvriers locaux, permettant par cette approche d'éviter le risque de réifier les significations des lois.

L'argument initial de Brubaker (1992), selon lequel le jus soli et le jus sanguinis sont liés à des conceptions profondes de type civique ou ethnique de l'appartenance, a été critiqué par la suite. D'autres analyses (Weil et Hansen, 1999 ; Joppke, 2003) ont montré la variabilité, même dans de courtes périodes de temps, de la législation sur la nationalité, et Weil (2002) en particulier a argumenté sur le fait que la parution du jus soli et du jus sanguinis en France et en Allemagne était justifiée par des objectifs contingents de gestion de l'immigration et par des choix de juristes, plutôt que par des conceptions de la nation. Brubaker lui-même (1999) a nié ouvertement l'existence de nations civiques ou ethniques.

La loi italienne sur la nationalité a été considérée par plusieurs commentateurs (Zincone, 2006a ; Colombo, Domaneschi et Marchetti, 2009 ; Zincone et Basili, 2013) comme un cas typique de loi ethnique, en entendant par «ethnique» une loi favorable aux descendants de citoyens et

\footnotetext{
${ }^{1} \mathrm{Si}$ dans le contexte français « nationalité » désigne le lien juridique entre l'individu et l'État, et « citoyenneté » désigne les droits, surtout politiques, liés à ce dernier (Ribert, 2006), en Italie, « cittadinanza » recouvre les deux acceptions (Sredanovic, 2014a ; cf. Trucco, 2015). Dans ce texte j’utilise le terme « nationalité » pour désigner le lien juridique et " citoyenneté/nationalité » dans les cas où les deux concepts sont mêlés.
} 
défavorable aux autres résidents. La loi, en vigueur depuis 1992, est relativement restrictive en regard de celles des autres pays membres de l'Union Européenne : les candidats ordinaires doivent justifier de dix ans de résidence continue (réduits à cinq ans pour les apatrides, à quatre pour les citoyens de l'UE) pour pouvoir entamer une procédure discrétionnaire de naturalisation. De plus, bien que les personnes nées en Italie aient droit à la nationalité italienne, ce droit ne s'exerce qu'à partir de 18 ans, et seulement dans le cadre d'une résidence continue. Par contre, la législation en Italie concède des droits significatifs de nationalité/citoyenneté aux émigrés, avec des mesures pour permettre à leurs descendants, même éloignés, d'obtenir la nationalité ; elle prévoit, en outre, le droit de vote à distance et une représentation spécifique de la « diaspora » au Parlement.

La récente analyse approfondie de Dumbrava (2014) nous permet de pousser plus loin l'examen des aspects ethniques (ou non) des lois sur la nationalité. Dumbrava ne se contente pas d'identifier la nature ethnique d'une loi avec sa clôture vis-à-vis des naturalisations ou du jus soli. L'auteur fait une distinction entre les normes qui facilitent la naturalisation de groupes ethniques spécifiques en tant que tels, ou de personnes avec des caractéristiques culturelles spécifiques, et les normes qui facilitent l'accès à la nationalité des anciens citoyens et de leurs descendants, ou des candidats ressortissants de territoires spécifiques. En outre, Dumbrava inclut aussi parmi les normes ethniques les asymétries dans la tolérance de la double nationalité (comme permettre aux nationaux italiens d'acquérir une autre nationalité, mais demander aux naturalisés de renoncer à leur nationalité d'origine), et la discrétion concernant les décisions d'attribuer la naturalisation (qui permet d'appliquer des critères ethniques). Or, si on utilise le terme « ethnique » pour caractériser une loi plus favorable aux émigrés qu'aux immigrés (cf. Joppke, 2003), la loi italienne s'inscrit clairement dans cette définition. On peut cependant aller plus loin, en s'appuyant sur l'analyse de Dumbrava, en réservant le terme «ethnique» aux cas où la nationalité est attribuée sur la base de la reconnaissance de l'appartenance à un groupe ethnique (voir infra pour les cas Allemand et Hongrois), « culturel » pour les réquisits relatifs à la connaissance de la langue, de l'histoire, etc. nationales, et en considérant séparément les critères fondés sur la descendance (« familisme ») ou sur la résidence dans un territoire spécifique hors du territoire national ${ }^{2}$. Cette distinction fondée sur quatre critères permet de montrer que, si le critère de descendance est très fort dans la loi italienne, les critères territoriaux et ethniques ont un rôle respectivement marginal et très marginal, et le critère culturel, bien que présent, est moins développé que dans le reste de l'Union Européenne.

\footnotetext{
${ }^{2}$ Ces usages des termes « ethnique » et « culturel » sont évidemment encore approximatifs, si l'on considère la critique de l'ethnicité faite, par exemple, par Brubaker (2004), ou, en anthropologie, par Gallissot, Kilani et Rivera (2000). J'utilise ces approximations essentiellement pour reprendre, et chercher à mettre ordre, dans les termes du débat sur les lois relatives à la nationalité débuté avec Brubaker (1992).
} 
Dans ce texte, je combine l'analyse de la loi dans son évolution récente et sa forme actuelle avec l'analyse des propositions de loi de la période 1996-2015, ainsi qu'avec les données d'une recherche portant sur 60 entretiens approfondis, menés dans la province de Ferrare entre 2010 et 2011 avec des migrants de différentes provenances (Maroc, Ukraine, Liban et Jordanie/Palestine), des ouvriers locaux et des membres d'associations pro-migrants (dont une partie des résultats a déjà été publiée en Sredanovic, 2014a ; 2014b ; Della Puppa et Sredanovic, à paraitre).

Je commence l'analyse en présentant les origines de la loi de 1992 ; je détaille ensuite les motivations pour lesquelles la loi italienne, dans ses normes, peut être considérée comme « familiste » plutôt qu' « ethnique », en exposant les différences avec les cas Allemand et Hongrois, considérés comme des lois fondées plus spécifiquement sur la reconnaissance d'une appartenance ethnique. Je souligne toutefois l'existence d'une exception dans la loi italienne, dans laquelle des critères culturels et strictement ethniques existent, exception relative au cas de la population des « confins orientaux » avec l'Autriche, la Slovénie et la Croatie. Je poursuis avec l'analyse du débat législatif sur l'introduction de critères culturels pour la naturalisation. Enfin, je montre comment les conceptions quotidiennes mises en lumière par les entretiens, bien que différentes de celles présentes dans la loi, manifestent également une réticence à accepter les critères culturels pour l'attribution de la nationalité.

\section{La loi sur la nationalité en Italie après 1947}

Après les normes relatives aux transformations territoriales du Traité de Paris de 1947 (Donati, 2013, p. 246 à 254), l'activité législative en matière de nationalité en Italie fut essentiellement stationnaire pendant 40 ans : les innovations les plus importantes - la conservation de la nationalité pour les femmes mariées à des non nationaux (1977) et la parité de genre dans la transmission de la nationalité (1983) - sont dues à des sentences de la Cour Constitutionnelle et non à l'initiative parlementaire (Zincone, 2006b, p. 10 à 13 ; cf. Knop, 2001). Le débat fut aussi limité, et il semblerait que les juristes eux-mêmes considéraient la question de la nationalité/citoyenneté comme marginale. Bien entendu, la citoyenneté matérielle (Baglioni, 2015) était tout sauf équitablement distribuée parmi la population. Dans certains cas, cette inégalité avait aussi des sources juridiques. Les migrants internes de l'Italie du Sud, par exemple, rencontraient de grandes difficultés à obtenir la résidence légale (et donc l'accès à des contrats de travail réguliers) dans le Nord du pays, cela à cause d'une loi contre l'urbanisation datant de la période fasciste, abolie seulement en 1961 (Alasia et Montaldi, 1975 ; De Michele, 2012). En outre, les historiens (et certains sociologues de l'époque) 
ont démontré la stigmatisation des migrants internes, comme, par exemple, la pathologisation des enfants à l'école (De Michele, 2010 ; 2012).

Le parcours de la loi de 1992 a été initié avec la conférence sur l'émigration italienne de 1988 : Giulio Andreotti, à l'époque ministre des affaires étrangères, s'attachait à promouvoir une réforme de la nationalité en faveur des émigrés et de leurs descendants (Pastore, 1999). Ce processus se base sur la même approche, d'orientation envers les émigrés, que celle de la loi précédente de 1912, avec laquelle le gouvernement cherchait à la fois à améliorer les conditions des émigrés et à renforcer leur rapport avec le pays d'origine, pour des questions de transferts d'épargnes, et dans l'espoir de transformer les émigres en lobbies pour la politique étrangère (Tintori, 2006 ; 2009a). Si la loi de 1912 a été introduite pendant le plus important flux d'émigration de l'histoire Italienne, la loi de 1992 a été introduite non seulement après une longue période de faible intérêt pour la nationalité, mais aussi lors d'une baisse de l'émigration. Cette émigration, encore très significative dans les années 1950 et 1960, voyait moins de 100000 départs par an en 1975 déjà, et elle a atteint son minimum dans la deuxième moitié des années 1990 (Einaudi, 2007, p. 52 à 57 - mais l'émigration italienne a connu une nouvelle hausse importante, avec un record de 311000 en 2010). En fait, on pourrait avancer l'hypothèse que la baisse de l'émigration ait même favorisé la capacité d'influence des émigrés sur la politique italienne : les organismes diplomatiques tout comme les associations étaient moins mobilisées sur le front des nouvelles arrivées et pouvaient s'occuper de questions comme celle touchant à la nationalité.

La loi fut débattue au Parlement en 1992, pendant les derniers jours d'activité précédant les élections qui eurent lieu cette année-là, et manqua donc de véritable discussion politique. Le fait de la présenter comme une loi en faveur des émigrants, a permis de faire oublier qu'elle faisait passer de 5 à 10 ans le réquisit de résidence en Italie des candidats à la naturalisation ne provenant pas de l'UE. Le rapporteur Mazzola (Démocratie Chrétienne) définissait les migrants ne provenant pas de l'UE comme un « groupe résiduel » (Pastore, 1999), bien que 77\% des non nationaux en Italie à l'époque (275.354 sur 356.159) provenaient d'un pays hors UE. Le vote fut donc unanime, et les médias accordèrent très peu d'attention à cette réforme.

\section{2. « Ethnique » ou « familiste »?}

La loi de 1992 est donc une loi pensée surtout pour les descendants des nationaux italiens émigrés, et elle a été approuvée alors que la conscience de l'immigration en Italie était encore récente (Einaudi, 2007) et pour cela, les effets de la loi sur les immigrés en Italie ont été négligés. À 
partir de la fin des années 90, le débat politique et les propositions de loi ont considéré la possibilité de réformer les conditions de naturalisation et du jus soli, mais l'absence d'une majorité parlementaire sur le sujet a bloqué presque complètement les réformes dans cette direction. Au contraire, les normes pour la transmission et la ré-acquisition de la nationalité des émigrés ont été réformées en 1994, 1996, 2000 et 2006.

Si cette forte attention des législateurs pour les émigrés (comparée à celle accordée aux immigrés) a amené les commentateurs académiques à qualifier la loi d'« ethnique », Zincone (2006b) en particulier a aussi qualifié cette loi de «familiste », en raison des conditions favorables aux descendants des nationaux mais aussi en référence aux réquisits limités, en 2006, pour obtenir la naturalisation par mariage ${ }^{3}$. Toutefois, ces derniers réquisits - six mois de résidence en Italie avant le mariage - ont été modifiés en 2009, et aujourd'hui le candidat doit avoir été marié pendant deux ans avant de pouvoir demander une naturalisation.

Malgré ce changement, le concept de «familisme» est particulièrement approprié pour la loi italienne : avec une exception sur laquelle je reviendrai dans le paragraphe suivant, les seuls critères d'attribution de la nationalité aux émigrés sont la parentalité et la résidence historique - les critères territoriaux et de descendance mentionnés dans l'introduction. Avoir un parent ou un grand-parent national est une condition nécessaire et suffisante pour ces candidats pour demander la nationalité italienne. Tintori (2009b) a montré qu'une majorité des naturalisations par cette voie concerne le Brésil, l'Argentine et l'Uruguay (66\% des naturalisations en consulat entre 1998 et 2007 ont été effectuées au Brésil et en Argentine), et qu'une partie significative des naturalisés n'ont pas de liens culturels ou sociaux avec l'Italie.

Même dans le cas des naturalisations liées à la résidence et à la naissance, les critères culturels sont réduits : les candidats nés en Italie sont citoyens de droit s'ils remplissent la condition de résidence régulière et continue, et la lettre de la loi est silencieuse sur les critères culturels nécessaires à leur naturalisation pour résidence, bien que cette dernière soit discrétionnaire. Dans ce dernier cas, les normes sont confinées à une circulaire ministérielle (K.60.1/86 du 1997) qui demande aux autorités de police d'interviewer les candidats pour relever leur niveau d'intégration et de compétence linguistique et transmettre une opinion au Ministère où la décision finale est prise. L'application de cette circulaire est très variable dans les différentes provinces italiennes, et la simple opinion est peu significative dans le contexte de la diffusion des tests de naturalisation dans les pays de l'Union Européenne (van Oers, Ersbøll et Kostakopoulou, 2010). Paradoxalement,

\footnotetext{
${ }^{3}$ La référence au familisme est un écho clair du « familisme amoral » de Banfield (1958), mais Zincone ne fait aucune référence explicite à l'œuvre de Banfield, et définir comme familiste la loi sur la nationalité n'implique pas d'adhérer à l'idée que la culture italienne exclurait toutes les solidarités au-delà de la famille. 
depuis 2010, les migrants qui demandent un permis de séjour de longue durée sont soumis à un test de compétences linguistiques, et un parcours d'intégration obligatoire a été introduit pour certains nouveaux arrivants en 2012 (voir Russo Spena et Carbone, 2014). Par contre, l'immobilisme de la législation sur la nationalité italienne, maintient d'une part le réquisit de résidence à des niveaux prohibitifs, et évite de l'autre les tendances restrictives fondées sur les tests.

Une brève comparaison avec les cas allemand et hongrois serait utile pour mettre l'accent sur le contraste qui existe entre ce type de normes et celles plus strictement ethnoculturelles. Dans le cas allemand, il existe le statut de Aussiedlers - désignant les Allemands ethniques ressortissants de pays communistes et, après 1989, exclusivement de l'ex-Union Soviétique - lequel permet d'obtenir la nationalité immédiatement après l'installation en Allemagne (Hailbronner et Farahat, 2015). Les conditions d'attribution du statut d'Aussiedler ont varié, mais le critère a toujours été la démonstration, par plusieurs moyens, y compris un test linguistique obligatoire à partir de 1996, d'une ethnicité allemande par descendance, éducation et culture. Des auteurs (Klekowski von Koppenfels, 2002; Hansen, 2003) ont affirmé que cette norme n'est pas le résultat d'une conception exclusivement ethnique de la nationalité/citoyenneté en Allemagne, mais plutôt d'objectifs politiques vis-à-vis de la République Démocratique Allemande et du Bloc Oriental en général ; toutefois, cette approche, qui prévoit différentes formes de documentation, est éloignée du principe de descendance propre à la loi italienne. De façon similaire, la loi hongroise reconnaît le statut de «Hongrois ethnique » (Kovács et Tóth, 2013) qui, bien que fondé principalement sur la descendance d'un national hongrois, prend également en considération une compétence culturelle (et, dans le passé, la certification était attribuée par des associations hongroises). Ce statut donne droit à une naturalisation accélérée, mais aussi à des droits sociaux même sans naturalisation.

L'approche strictement ethnoculturelle est plus difficile à trouver dans la législation italienne, sauf dans un cas particulier qui fera l'objet du prochain paragraphe.

\section{L'exception : la « frontière orientale »}

Le seul cas où la loi italienne reconnaît l'importance de la culture, et même d'une ethnicité stricto sensu, italienne, en référence à des questions de nationalité, est celui des habitants des territoires transférés de l'Autriche-Hongrie à l'Italie après la Première Guerre mondiale (TrentinTyrol du Sud, Gorizia, Trieste, et Istrie et Dalmatie septentrionale, rattachés à la Yougoslavie après la Deuxième Guerre mondiale) et de leurs descendants. En particulier, la loi 379 de 2000 a concerné les descendants de ceux qui, émigrés de ces territoires avant leur annexion à l'Italie avec le Traité 
de Saint-Germain-en-Laye de 1919 et le Traité de Rapallo de 1920, ont fait une demande de naturalisation entre 2000 et 2005 et ont pu démontrer une appartenance culturelle et ethnique à l'Italie. Il s'agissait donc de descendants de «nationaux manqués », et les travaux préparatoires montrent qu'à l'origine de la loi il y a eu une pétition de descendants d'émigrés du Trentin au Brésil. En absence du critère de nationalité des parents/grands-parents, la circulaire ministérielle faisait référence à un droit d'option à la nationalité italienne contenu dans le Traité de SaintGermain-en-Laye, et prévoyait, outre les documents attestant la résidence dans ces territoires et l'émigration avant 1920, toute documentation, tant familiale que fournie par les associations italiennes du lieu de résidence, qui démontrait l'appartenance au groupe ethnolinguistique (ce dernier correspond donc au critère ethnique stricto sensu).

Le même schéma a été répété dans la réforme de 2006, concernant les ex-nationaux italiens (et leurs descendants) résidant dans les territoires transférés de l'Italie à la Yougoslavie après la Deuxième Guerre mondiale, ayant perdu la nationalité lors de cette transition. Même si dans ce cas la loi existante semblait appropriée, les conditions de la loi de 2000 s'appliquent aussi à ces candidats, puisque tant le Traité de Paris de 1947 que celui d'Osimo de 1975, permettaient l'option pour la nationalité italienne seulement en cas d'appartenance linguistique et ethnique à l'Italie.

Ces exceptions relativement marginales, dans lesquelles la loi italienne revêt aussi une dimension ethnique et culturelle au sens strict, sont donc liées à des traités internationaux, qui constituaient dans une certaine mesure une source juridique extérieure au système juridique italien. Donati (2013, p. 246 à 254) a toutefois souligné que la Constitution de 1948, dans l'article 51 sur les services publics, prévoyait déjà la possibilité d'accès aux fonctions publiques aux «Italiens qui n'appartiennent pas à la République », et propose qu'il s'agisse d'une reprise du concept d' « italiens non régnicoles » avec lequel l'Italie monarchique facilitait la naturalisation des membres des populations envers lesquelles elle menait une politique irrédentiste. En outre, il faut souligner que la loi de 2006 s'inscrit dans le cadre d'une des plus importantes politiques de la mémoire et des minorités des 20 dernières années en Italie. Les travaux préparatoires montrent des liens forts avec la politique qui a fait des minorités italiennes en Slovénie et Croatie une question nationale et de la mémoire, et a conduit, en 2004, à l'introduction du « Jour du souvenir », sur le modèle du Jour de la mémoire des victimes de l'Holocauste, dans l'anniversaire du Traité de Paris.

\section{Les propositions de loi}


Si la loi sur la nationalité en Italie est encore en large partie celle de 1992, un très ample débat a été mené sur la question. Entre 1996 et 2016, 153 propositions de loi ont été présentées. Ce débat a été largement improductif, car, au-delà des normes sur les émigrés, la modification des réquisits pour obtenir la naturalisation par mariage a été la seule modification significative approuvée. Toutefois, l'analyse des nombreuses propositions de loi peut éclairer sur les attitudes des législateurs.

55 propositions (plus d'un tiers des 153 présentées), dont 46 de façon exclusive, portaient sur les questions de la nationalité des émigrés italiens et de leurs descendants, et de la ré-acquisition de la nationalité. Il faut aussi souligner que depuis 2006, une partie des parlementaires sont élus exclusivement par les citoyens italiens résidant à l'étranger, et que ces parlementaires sont particulièrement engagés sur ce thème, conscients que les naturalisations à l'étranger peuvent potentiellement augmenter leur électorat. 26 autres propositions ont porté exclusivement sur le jus soli, qui est devenu la question centrale du débat à partir de 2012 (Sredanovic et Farina, 2015). Ces propositions ont remporté un certain succès, puisqu'une réforme introduisant des éléments de jus soli à la naissance a été votée par la Chambre des députés fin 2015. Toutefois, au moment de la rédaction du présent texte, la proposition de loi (S.2092/XVII de 2015) est bloquée au Sénat, et le Gouvernement Renzi a été le quatrième gouvernement de centre-gauche, de 2000 à aujourd'hui, à entamer une réforme de la loi sur la nationalité sans parvenir à la voter.

Un petit nombre de propositions de loi (7) est orienté vers des groupes ethniques, nationaux ou religieux spécifiques. Deux propositions de loi (dont une présentée dans trois législatures différentes) comptent parmi les rares cas où le passé colonial italien est abordé dans le débat public italien. La proposition C.5634/XIII de 1999 demandait la résolution de la situation des enfants de pères italiens et de mères érythréennes, que les lois raciales interdisaient aux pères de reconnaître ; une deuxième (C.6091/XIV de 2005), de reconnaître la nationalité aux élèves somaliens des écoles militaires italiennes. Deux autres propositions avaient pour but de faciliter l'accès à la nationalité respectivement aux membres de l'Union des Communautés Hébraïques en Italie (S.730/XV de 2006) et aux descendants de la diaspora hébraïque italienne de 1492 (C.3291/XVII de 2015), options qui rappellent les mesures en faveur de la naturalisation des juifs séfarades en Espagne.

Parmi les 59 propositions de loi ayant pour objet une réforme plus large de la nationalité, et en particulier la réforme de la naturalisation ordinaire, 36 incluaient un test de naturalisation, souvent couplé avec une réduction des réquisits de résidence. Dans la version la plus répandue, ce test devrait vérifier la connaissance de la langue italienne, de la « vie civile » en Italie, de l'éducation civique et de la Constitution. Certaines variations existent, car dans certains cas la proposition de 
test de naturalisation ne s'accompagne pas d'une réduction des réquisits de résidence : c'est le cas de la proposition C.2376/XVII de 2014, ou le réquisit devient un cours annuel obligatoire avec un test final. La Ligue du Nord a proposé plusieurs fois (la plus récente est la S.2394/XVII de 2016) une modification de la loi qui, sans toucher aux réquisits de résidence, aurait rendu nécessaire aussi la connaissance de l'histoire et de la culture italiennes, des institutions, même locales, et des langues régionales reconnues (principalement celles des minorités linguistiques frontalières et isolées, outre le Frioulan et le Sarde). Bien que la Ligue du Nord soit généralement le parti le plus restrictif envers les immigrés, dans ces cas, il n'a pas proposé de réquisits de résidence plus sévères, initiative prise par des parlementaires proche de Forza Italia. C'est le cas, par exemple, de la proposition S.1765/XVI de 2009, selon laquelle un test sur la connaissance des langue, institutions et traditions, même religieuses, italiennes, encadré par l'Arme des Carabiniers, devrait avoir lieu après 8 ans de résidence, et ne donnerait le droit de vote qu'aux élections locales et celui de demander la naturalisation qu'après 12 ans de résidence.

Tous les initiatives d'introduction des tests sont liées au concept d'intégration, qui, dans certains des textes proposés, devrait être vérifié sur le plan social autant que culturel. En revanche, l'idée d'un test destiné aux descendants des émigrés italiens n'apparaît que dans deux propositions de loi. Tintori (2009b) souligne combien, dans le cas des descendants d'émigrés, l'intégration est considérée comme allant de soi, au point que certaines régions italiennes ont promu des programmes pour attirer ces membres de la « diaspora», en tant qu'alternative aux immigrés économiques. L'auteur critique les résultats effectifs de ces programmes, et, plus généralement, les normes sur la nationalité des descendants des émigrés, en soulignant qu'une partie considérable des naturalisés en tant que descendants de nationaux sont exclusivement hispanophones ou lusophones et qu'ils sont plus intéressés par l'obtention d'un passeport pour l'Espagne ou les États-Unis que par la perspective de s'installer en Italie.

\section{La citoyenneté quotidienne}

Jusqu'à présent, on a présenté la définition légale de la nationalité/citoyenneté en Italie, mais il faut souligner qu'il est inapproprié d'attribuer des conceptions sous-jacentes à une culture nationale, ou aux visions de la population, parce que ces dernières ont une autonomie, et peuvent être très différentes les unes des autres (Miller-Idriss, 2006). Dans ce sens, l'approche de la citoyenneté quotidienne, l'étude qualitative des visions de la nationalité/citoyenneté de la population générale, peut contribuer à la compréhension des conceptions de citoyenneté existantes dans la société au- 
delà des lois et du débat parlementaire. Duchesne (1997) a par exemple montré la coprésence d'individualistes-universalistes et de nationaux-territoriaux en France, et Venel (2004) et Ribert (2006) ont montré la variété d'identifications possibles entre, respectivement, identification avec la France et avec la religion musulmane, et entre la France et le pays des parents.

Dans ma recherche, menée à Ferrare, sur les conceptions de la nationalité/citoyenneté de migrants, ouvriers locaux et membres d'associations pro-migrants, je me suis focalisé, plutôt que sur les principes d'identification, sur les conditions considérées comme équitables pour l'obtention de la nationalité/citoyenneté italienne (voir les recherches similaires de Miller-Idriss 2006; Colombo 2009, 2010). En suivant l'approche en particulier de Duchesne (1997) et de Venel (2004), j'ai individué des axes principaux avec lesquels identifier les positions idéal-typiques parmi mes interviewés (Sredanovic, 2014b) La majorité des interviewés s'est exprimée en faveur d'un renforcement du jus soli. En outre, une conception économique de la nationalité/citoyenneté, fondée sur le fait de travailler et de payer des impôts, plutôt que sur la résidence ou sur la culture, était prévalente parmi les interviewés, et surtout chez les ouvriers locaux (Sredanovic, 2014b). Si ces réquisits sont bien présents dans la loi italienne, il y a clairement une distance entre les orientations des interviewés et à la fois la loi actuelle et le débat parlementaire.

Ces données sont bien obtenues par une recherche qualitative incluant uniquement des ouvriers parmi les autochtones interviewés, dans un territoire historiquement ancré à gauche comme celui de Ferrare. Cependant, les résultats de la recherche de Colombo $(2009$; 2010) avec des étudiants de Milan - zone beaucoup moins à gauche - montrent une méfiance similaire envers les réquisits culturels et définissent le citoyen comme individu respectant les lois et payant des impôts ${ }^{4}$. Ces recherches qualitatives ouvrent donc aussi sur des réflexions sur l'identité nationale en Italie. Isernia (2003) affirmait que l'identité italienne est ethnique plutôt que civique, car les résultats des surveys indiquent une confiance réduite dans les institutions et une forte identification à la nationalité. Si on pose la question en termes d'accès à la communauté plutôt qu'en termes d'identité personnelle, les participants à ma recherche ne semblent pas exiger une conformité culturelle, mais plutôt une participation économique des nouveaux citoyens ${ }^{5}$. Une motivation possible de cette orientation peut être trouvée dans le travail de Patriarca (2010) sur les discours politiques sur le caractère italien. La chercheuse a montré la reproduction continue de certains auto-stéréotypes au cours des deux

\footnotetext{
${ }^{4}$ Parmi les interviewés autochtones de Colombo on trouve cependant une minorité hostile à l'Islam, position absente parmi les interviewés dans ma recherche.

${ }^{5}$ Des enquêtes ont inclus des questions sur les conditions que les immigrés devraient remplir avant la naturalisation (Brandi, Caruso et Cerbara, 2010 ; Transatlantic Trends 2010). Cependant, ces enquêtes n'ont pas inclus la participation économique parmi les réponses possibles. En l'absence de cette option, les interviewés de Brandi, Caruso et Cerbara se sont focalisés sur le partage des traditions (qui est resté très marginal dans mes entretiens) et ceux de Transatlantic Trends sur le respect de la loi (qui est apparu aussi dans ma recherche). Voir Sredanovic, 2012, pp. 105-114 pour une synthèse plus ample.
} 
derniers siècles et demi, malgré les profonds changements sociaux et institutionnels du pays : nation inachevée, population familiste et ingouvernable, capacité innée de se débrouiller mais incapacité d'organisation, etc. Autrement dit, même si les Italiens peuvent éprouver une identification individuelle avec le pays fondée sur des critères culturels ou de descendance (plutôt que sur l'acceptation des normes juridiques essentielles), il est probable que chacun considère cette identification comme limitée à une minorité de la population. Plusieurs interviewés ont fait référence à l'un des auto-stéréotypes analysés par Patriarca quand je leur demandais s'ils auraient trouvé juste d'introduire des tests de langue, culture et législation pour les candidats à la naturalisation. Considérant qu'une partie significative de la population italienne native ne maîtrise pas cette connaissance de la culture italienne, ces interviewés trouvaient injuste de demander aux candidats à la naturalisation des réquisits qui ne seraient pas remplis par des Italiens de naissance.

Il est aussi intéressant de noter que parmi la minorité des participants qui considéraient les tests comme positifs, plusieurs visions globales différenciées s'exprimaient : pour certains, cet aspect s'intégrait au devoir des immigrés de se conformer à la culture locale, pour d'autres interviewés ces tests participaient d'une politique active d'intégration.

\section{Conclusion}

La nationalité italienne change donc de profil selon l'angle d'analyse. Les normes de la loi sont conçues pour les descendants des émigrés plutôt que pour les immigrés, et l'activité législative post1992 s'est également concentrée sur les émigrés. Cependant, en suivant la typologie de Dumbrava (2014), on peut dire que les normes qui visent de façon spécifique des groupes ethniques - et même des normes culturelles - ont un rôle plutôt limité dans le texte de la loi. Si on étend l'analyse aux propositions de loi, on peut voir une orientation « culturaliste » du débat, car l'introduction de tests de naturalisation a été proposée plusieurs fois au Parlement (même le débat dans les médias sur le jus soli a été caractérisé par l'insistance sur la culture - voir Sredanovic et Farina, 2015). Cependant, la difficulté à légiférer sur la nationalité a empêché toute modification de la loi dans ce sens. En outre, l'« italianité » des émigrés et de leurs descendants ne semble pas être mise en cause et l'idée de vérifier les compétences linguistique et culturelle de ces candidats à la nationalité est très éloignée.

Les entretiens recueillis à Ferrare, bien que limités à des ouvriers et menés dans une zone marquée politiquement, expriment une méfiance envers les conceptions culturelles de la nationalité, et en particulier envers les tests de naturalisation. D'ailleurs, si le familisme semble être une 
synthèse efficace pour la loi sur la nationalité, les interviewés semblent être plutôt orientés sur des conceptions économiques de la nationalité/citoyenneté.

Cette analyse permet de complexifier l'idée que la citoyenneté/nationalité et l'identité nationale italienne soient « ethniques ». La recherche basée sur la citoyenneté quotidienne suggère plutôt des principes d'appartenance éloignés d'une dimension culturelle. La loi sur la nationalité est sûrement restrictive pour les immigrés si on la compare aux lois d'autres pays de l'Union Européenne, mais restrictive et « ethnique » ne sont pas des synonymes (Dumbrava, 2014). En fait, on a vu comment les critères strictement ethniques, et même culturels, sont limités dans la loi actuelle, et si les tests de naturalisation ont été proposés plusieurs fois au Parlement, les normes visant des groupes ethniques au-delà de la descendance restent marginales dans le débat italien.

\section{Références bibliographiques}

Alasia F., Montaldi D. (1975), Milano, Corea: inchiesta sugli immigrati, Milano, Feltrinelli.

Baglioni L. G. (2015), «La citoyenneté matérielle : définition et analyse comparative FranceItalie », Pôle Sud, $\mathrm{n}^{\circ} 43$.

Banfield E. C. (1958), The Moral Basis of a Backward Society, Glencoe, Il., Free Press.

Brandi M. C., Caruso M. G., Cerbara L. (2010), « Le opinioni degli italiani sulle migrazioni qualificate dall’Est Europa », Studi Emigrazione, $\mathrm{n}^{\circ} 179$.

Brubaker R. (1992), Citizenship and Nationhood in France and Germany, Cambridge, Ma., Harvard University Press.

Brubaker R. (1999), «The Manichean Myth: Rethinking the Distinction between «Civic» and «Ethnic» Nationalism », in Kriesi H., Armingeon K., Siegrist H., Wimmer A. (eds.) Nation and National Identity. The European Experience in Perspective, Chur, Rüegger.

Brubaker R. (2004), Ethnicity without Groups, Cambridge, Ma., Harvard University Press.

Colombo, E. (2009), «Oltre la cittadinanza multiculturale. La rappresentazione dei diritti, dei doveri e delle appartenenze tra alcuni giovani delle scuole superiori », Rassegna italiana di sociologia, vol. $50, \mathrm{n}^{\mathrm{o}} 3$.

Colombo, E. (2010), « Changing citizenship: everyday representations of membership, belonging and identification among Italian senior secondary school students », Italian Journal of Sociology of Education, vol. 2, no 1 .

Colombo E., Domaneschi L., Marchetti C. (2009), Una nuova generazione di italiani. L'idea di cittadinanza tra i giovani figli di immigrati, Milano, FrancoAngeli. 
Della Puppa F., Sredanovic D. (à paraître), « Citizen to Stay or Citizen to Go? Naturalization, Security, and Mobility of Migrants in Italy », Journal of Immigrant \& Refugee Studies, doi : http://dx.doi.org/10.1080/15562948.2016.1208316

De Michele G. (2010), « "Un'inguaribile incapacità di reazione al nuovo ambiente di vita". Bambini meridionali al Nord e classi differenziali negli anni '50 e '60 », Rivista sperimentale di freniatria, vol. 134, n 3 .

De Michele G. (2012), 'At the gates of civilization'. Southern children in Turin primary schools, 1950s-1970s, thèse doctorale, University of Reading.

Donati S. (2013), A Political History of National Citizenship and Identity in Italy, 1861-1950, Stanford, Stanford University Press.

Duchesne S. (1997), Citoyenneté à la française, Paris, Presses de SciencesPo.

Dumbrava C. (2014), Nationality, Citizenship and Ethno-cultural Belonging. Preferential Membership Policies in Europe, Basingstoke, Palgrave Macmillan.

Einaudi L. (2007), Le politiche dell'immigrazione in Italia dall'Unità a oggi, Roma-Bari, Laterza.

Gallissot R., Kilani M., Rivera A. (2000), L’imbroglio ethnique en quatorze mots clés, Lausanne, Payot.

Hailbronner K., Farahat A. (2012), Country Report : Germany, EUDO Citizenship, http://cadmus.eui.eu/bitstream/handle/1814/34478/EUDO_CIT_2015 02-Germany.pdf? $\underline{\text { sequence }=1}$

Hansen R. (2003), « Citizenship and Integration in Europe », in Joppke C., Morawska E. (eds.) Toward Assimilation and Citizenship. Immigrants in Liberal Nation-States, Houndmills, Palgrave-Macmillian.

Isernia P. (2003), « Changement et continuité dans la culture politique italienne », Pôle Sud, nº 19.

Joppke C. (2003), « Citizenship between De- and Re- Ethnicization », Archives Européennes de Sociologie, vol. 44, no 3 .

Klekowski von Koppenfels A. (2002), « Politically Minded : The Case of the Aussiedler as an Ideologically Defined Category », in Hunger U., Meendermann K., Santel B., Woyke W. (eds.), Migration in erklärten und 'unerklärten' Einwanderungsländern: Analyse und Vergleich, Münster, Lit Verlag.

Knop K. (2001), « Relational Nationality: On Gender and Nationality in International Law », in Aleinikoff T. A., Klusmeyer, D. (eds.) Citizenship Today. Global Perspectives and Practices, Washington, D.C., Carnegie Endowment for International Peace. 
Kovács M. M., Tóth J. (2013), Country Report : Hungary, EUDO Citizenship, http://eudocitizenship.eu/admin/?p=file\&appl=countryProfiles \&f=2013-18-Hungary.pdf

Miller-Idriss C. (2006), " Everyday Understandings of Citizenship in Germany », Citizenship Studies, vol. $10, \mathrm{n}^{\mathrm{0}} 5$.

Pastore F. (1999), « Droit de la nationalité et migrations internationales : le cas italien », in Weil P., Hansen, R. (eds.) Nationalité et citoyenneté en Europe, Paris, La Decouverte.

Patriarca S. (2010), Italian Vices. Nation and Character from the Risorgimento to the Republic, Cambridge, Cambridge University Press.

Ribert É. (2006), Liberté, égalité, carte d'identité: les jeunes issus de l'immigration et l'appartenance nationale, Paris, La Decouverte.

Russo Spena M., Carbone V. (eds.) (2014), Il dovere di integrarsi. Cittadinanze oltre il logos multiculturalista, Roma, Armando.

Sredanovic D. (2012), Diventare cittadini, rimanere cittadini. Concezioni della cittadinanza e biografie di diritti di migranti e operai locali a Ferrara, thèse de doctorat, Università di Padova.

Sredanovic D. (2014a), « Quelle est la valeur de la nationalité/citoyenneté en Italie ? Résultats d'une recherche auprès des migrants et des ouvriers italiens à Ferrare », Migrations Société, nº 153154.

Sredanovic D. (2014b), « Culture or taxes ? The conceptions of citizenship of migrants and local factory workers in Italy », Citizenship Studies, vol. 18, $\mathrm{n}^{\circ}$ 6-7.

Sredanovic D., Farina F.G. (2015), «Can Youth with a Migrant Background Speak? Representation, Citizenship and Voice in Italian TV and Press Journalism », Journal of Intercultural Studies, vol. $36, \mathrm{n}^{\circ} 6$.

Tintori G. (2006), «Cittadinanza e politiche di emigrazione nell'Italia liberale e fascista. Un approfondimento storico », in Zincone G. (dir.) Familismo legale. Come (non) diventare italiani, Roma-Bari, Laterza.

Tintori G. (2009a), « Nuovi italiani e italiani nel mondo. Il nodo della cittadinanza », in Corti P., Sanfilippo M. (dir.) Storia d'Italia. Annali 24. Migrazioni, Torino, Einaudi.

Tintori G. (2009b), Fardelli d'Italia ? Conseguenze nazionali e transnazionali delle politiche di cittadinanza italiane, Roma, Carocci.

Transatlantic Trends (2010), Transatlantic Trends Immigration, Topline Data 2010 report, http://rends.gmfus.org/files/2011/12/TTI2011_Topline_final1.pdf

Trucco D. (2015), « Jeunes, musulmans et italiens (ou presque). Représentations de la citoyenneté et rapport au politique d'un groupe d'enfants d'immigrés à Gênes », Pôle Sud, no 43. 
van Oers R., Ersbøll E., Kostakopoulou T. (eds.) (2010), A Re-definition of Belonging? Language and Integration Tests in Europe, Leiden, Martin Nijhoff.

Venel N. (2004), Musulmans et citoyens, Paris, PUF.

Weil P. (2002), Qu'est-ce qu'un Français? Histoire de la nationalité depuis la Révolution, Paris, Grasset.

Weil P., Hansen, R. (dir.) (1999), Nationalité et citoyenneté en Europe, Paris, La Decouverte.

Zincone G. (dir.) (2006a), Familismo legale. Come (non) diventare italiani, Roma-Bari, Laterza.

Zincone G. (2006b), « Due pesi e due misure : pronipoti d'Italia e nuovi immigrati », in Id. (dir.) Familismo legale. Come (non) diventare italiani, Roma-Bari, Laterza.

Zincone G., Basili M. (2013), Country Report: Italy, EUDO Citizenship, http://www.eudocitizenship.eu/images/docs/Italy_Citizenship_Report_update_2013\%20AMENDED.pdf 